\title{
ON HOMEOMORPHISMS OF INFINITE-DIMENSIONAL BUNDLES. I
}

BY

\author{
RAYMOND Y. T. WONG(1)
}

\begin{abstract}
In this paper we present several aspects of homeomorphism theory in the setting of fibre bundles modeled on separable infinite-dimensional Hilber $t$ (Fréchet) spaces. We study (homotopic) negligibility of subsets, separation of sets, characterization of subsets of infinite-deficiency and extending homeomorphisms; in an essential way they generalize previously known results for manifolds. An important tool is a lemma concerning the lifting of a map to the total space of a bundle whose image misses a certain closed subset presented as obstruction; from this we are able to obtain a result characterizing all subsets of infinite deficiency (for bundles) by their restriction to each fibre. Other results then follow more or less routinely by employing the rather standard methods of infinite-dimensional topology.
\end{abstract}

1. In this paper we extend the results on infinite-dimensional separable Hilbert spaces (manifolds) to fibre bundles modeled on them. The main results of this paper deal with bundles modeled on $s=(-1,1)^{\infty}$ (the countable infinite product of open intervals $(-1,1)$ ) and on the Hilbert cube $Q=[-1,1]^{\infty}$. (It is known that any separable infinite-dimensional Fréchet space is homeomorphic to $s$ [1].) In a later paper the author and Chapman [19] are able to use results of this paper to extend all the results to bundles modeled on $s$-manifolds, and in a certain appropriate manner, to bundles modeled on Q-manifolds [20].

A main result of this paper is the following characterization of projective $Z$-sets which then serve as an essential tool to obtain other results. We first need several conventions.

Fibre bundles $(E, p, B)$ are denoted by their total space $E$. Each bundle is defined to have a single fibre and for our purpose only the topological structure (local triviality) of a bundle is important. Bundle maps (or morphisms) and isomorphisms are defined as usual [12A] (isomorphisms are sometimes called bundle bomeomorphisms). In particular they will have the same base space $B$

Received by the editors November 18, 1970 and, in revised form, July 24, 1973. AMS (MOS) subject classifications (1970). Primary 57A20, 58B05.

Key words and phrases. Bundle, polyhedron, homeomorphism, ambient isotopy, deletion, separation, exten sion.

(1) This research is supported in part by NSF Grant GP 20632. 
and will be the identity on $B$. Bundle maps are also called $B$-preserving maps; in fact, we shall use such a term to describe a map from a subset $A$ of some product space $B \times X$ into another $B \times Y$ for which the map keeps the $B$-coordinate of each point unchanged. A bundle is trivial if it is isomorphic to a product bundle.

A polybedron is space homeomorphic $(\simeq)$ to $|K|$ where $K$ is a locally finite simplicial complex (Ifsc). A subset $A$ of a polyhedron $X$ is a subpolybedron in case there is a triangulation of pairs $(X, A) \rightarrow(|K|,|L|)$.

A closed subset $K$ of a space $X$ is a $Z$-set provided, for each nonempty homotopically trivial open set $U$ in $X, U \backslash K$ remains nonempty and homotopically trivial. A subset $A$ of a product space $X \times Y$ is an $X$-projective $Z$-set provided there is a closed $Z$-set $K \subset X$ for which $A \subset K \times Y$.

We say a space $X$ is $Y$-stable, for some space $Y$, if there is a homeomorphism of $X$ onto $X \times Y$. Suppose $X$ is $F$-stable, $F$ a homogeneous space, a subset $K \subset X$ is $F$-deficient if there is a homeomorphism $f: X \rightarrow X \times F$ such that $f(K) \subset X \times\{y\}$ for some point $y \in F$. Similarly we say $K \subset B \times X$ is F-deficient in bundle $B \times X=(B \times X, p, B)$ provided there is a $B$-preserving homeomorphism of $B \times X$ onto $B \times X \times F$ sending $K$ into $B \times X \times\{y\}$ for some point $y \in F$.

Hypothesis. All spaces, unless stated otherwise, are assumed metrizable.

Theorem 1.1 (characterization). Let $(B \times s, p, B)$ be a product bundle over polybedron $B$ and let $K C B \times s$ be closed. Then these are equivalent:

(A) $K \cap p^{-1}(b)$ is a Z-set in each $p^{-1}(b)$.

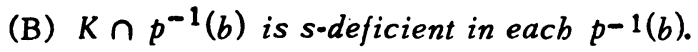

(C) $K$ is s-deficient in bundle $B \times s$.

(D) There is an isomorphism $\phi$ of $B \times s$ such that $\phi(K)$ is an s-projective Z-set.

Moreover, the same is true if $B$ is a retract of a polybedron.

Proof. $(A) \Leftrightarrow(B)$ is in [2]; (C) $\Leftrightarrow(D)$ is in [9A]. Obviously (C) or (D) implies (A).

The second half of Theorem 1.1 follows easily from the first. To see this let $B$ be a retraction of a polyhedron, say $P$, then let $r: P \rightarrow B$ be a retraction map. Let $\left(P \times s, p_{*}, P\right)$ be the pull-back induced by $r$. Let $r_{*}: P \times s \rightarrow B \times s$ denote the induced map and $K_{*}=r_{*}^{-1}(K)$. Then $K_{*}$ satisfies $(A)$ in bundle $P \times s$. By (D) there is a bundle isomorphism $\phi^{\prime}$ of $P \times s$ that sends $K_{*}$ onto an $s$-projective Z-set. Denote by $g$ the map $j \times$ id: $B \times s \rightarrow P \times s$, where $j$ is the inclusion. Then $\phi: B \times s \rightarrow B \times s$ defined by $\phi=\left.r_{*} \phi^{\prime}\right|_{g(B \times s)^{g}}$ is a required bundle isomorphism for (1).

The implication $(A) \Rightarrow(D)$ is an easy consequence of Theorem 3.1 and Lemma 3.4. Modulo these results we outline an argument in the following. It is 
known that $s \cong s \times Q[8 \mathrm{~A}]$. So we may replace $s$ by $s \times Q$. Write $Q \backslash s$ as a countable union of compact sets $K_{1}, K_{2}, \ldots$ Each $K_{i}$ is a $Z$-set in $Q$. Then each $L_{i}=B \times s \times K_{i}$ is an $(s \times Q)$-projective $Z$-set. By Theorem 3.1 there is an isomorphism $b$ of $E=B \times s \times Q$ such that $b(K) \cap\left(\bigcup_{i \geq 1} L_{i}\right)=\varnothing$. Hence $b(k) \subset$ $(B \times s) \times s$. By Lemma 3.4 we can push $b(K)$ further inside by another isomorphism $b_{1}$ so that $b_{1} b(K) \subset(B \times s) \times \prod_{i \geq 1}[-1 / 2,1 / 2]_{i}$. Let $\phi=b_{1} b$. Then $\phi(K)$ is an $(s \times Q)$-projective $Z$-set.

2. Lifting. We say a set $A \subset X$ is bomotopy negligible in $X$ provided the inclusion $j: X \backslash A \rightarrow X$ is a homotopy equivalence. (Note that, for manifolds, $Z$ sets are homotopy negligible.) Absolute retracts (AR) and absolute neighborhood retracts (ANR) are for metric spaces defined in the sense of Palais [14]. The following theorem also generalizes Lemma 4 of [18].

Theorem 2.1. Let $E=(E, p, B)$ be a bundle over Hausdorff space $B$ with fibre $F$ a metric $A R$. Let $A \subset E$ be closed and such that $A \cap p^{-1}(b)$ is bomotopy negligible in eacb $p^{-1}(b)$.

Suppose $(K, L)$ is any simplicial pair (weak topology) and $f$ is a map of $|K|$ into $B$. Then each lifting of $\|_{|L|}$ into $E \backslash A$ extends to one of $f$ into $E \backslash A$.

We remark that for $A=\varnothing$, Theorem 2.1 is well known [13, Theorem 9]. As an application we obtain the following corollaries (Corollary 2.3 generalizes Theorem 3 of [18]). The proof of Corollary 2.2 follows immediately from inspecting the proof of Theorem 2.1 .

Corollary 2.2. Let $(E, p, B)$ be a bundle over simplicial complex $B$ with fibre $F$ an ANR. Suppose $A \subset E$ is a closed set sucb that, for each $b \in B$, $p^{-1}(b) \backslash A$ is nonempty and bas the bomotopy type of a point; then $E$ admits a continuous cross-section $r: B \rightarrow E$ satisfying $r(B) \cap A=\varnothing$.

Define, for a closed subset $A$ of any space $X, A$ is strongly bomotopy negligible (SHN) if the inclusion $U \backslash A \rightarrow U$ is a homotopy equivalence for each open $U$ in $X ; A$ is locally bomotopy negligible $(\mathrm{LHN})$ if each point has a neighborhood system $\left\{U_{a}\right\}$ for which the inclusion $U_{a} \backslash A \rightarrow U_{a}$ is a homotopy equivalence. It is known (Eells-Kuiper [11]) that for metric ANR, SHN $\Leftrightarrow$ LHN. In the following we say $X$ is locally AR (respectively locally ANR) if each point has a neighborhood which is an AR (respectively ANR). It is known that a paracompact space which is locally ANR is an ANR. The following corollary identifies global negligible subsets by their restriction to the fibres. (Recall that all spaces involved are metrizable.)

Corollary 2.3. Let $E=(E, p, B)$ be a bundle over an ANR B with fibre a local $A R$ (or manifold). Then a closed set $A \subset E$ is strongly bomotopy negligible 
(respectively, a Z-set) if $A \cap p^{-1}(b)$ is locally bomotopy negligible (respectively, a Z-set) in eacb $p^{-1}(b)$.

Note that $E$ is an ANR and an ANR is necessarily locally pathwise connected. The above corollary follows routinely from Theorem 2.1 and EellsKuiper [11].

For a proof of Theorem 2.1 we need two lemmas. To state them we need some definitions. Let $\psi: X \rightarrow B$ be a map into the base space $B$ of a bundle $(E, p, B)$ and let $K \subset E, A \subset X$ be subsets. Then two liftings $\phi_{0}, \phi_{1}$ of $\psi$ into $E \backslash K$ are said to be $E$-bomotopic in $E \backslash K$ modulo $A$ provided $\phi_{0}$ and $\phi_{1}$ are joined by a homotopy $\left\{\phi_{t}\right\}$ of $X$ into $E \backslash K$ such that each $\phi_{t}$ lifts $\psi$ and, for all $t, r, \phi_{t}(x)=\phi_{r}(x)$ for any $x \in A$.

In the following let $A_{0}=[-1,0] \times J^{n}, A_{1}=[0,1] \times J^{n}$ where $J=[-1,1]$, $n \geq 0$, and let $A=A_{0} \cup A_{1}, A^{\prime}=A_{0} \cap A_{1}$.

Lemma 2.4. Let $(B \times F, p, B)$ be trivial over a Hausdorff space $B$ with fibre $F$ an absolute retract. Let $K$ be a closed subset of $E=B \times F$. Let $\phi$ : $A \rightarrow B$ be a map. Suppose, for $i=0,1, \phi_{i}: A_{i} \rightarrow E \backslash K$ are liftings over $\left.\phi\right|_{A_{i}}$,

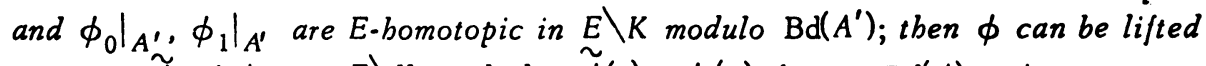
to a map $\tilde{\phi}$ of $A$ into $E \backslash K$ sucb that $\tilde{\phi}(x)=\phi_{i}(x)$ for $x \in \operatorname{Bd}(A) \cap A_{i}$

Proof. Let $\left\{b_{t}\right\}$ denote an $E$-homotopy in $E \backslash K$ modulo $\mathrm{Bd}\left(A^{\prime}\right)$ between $b_{0}=$ $\left.\phi_{0}\right|_{A^{\prime}}$ and $b_{1}=\left.\phi_{1}\right|_{A^{\prime}}$. Let $X=A \times[0,1]$ and define $\psi: X \rightarrow B$ by $\psi(x, t)=$ $\phi(x)$ for all $t$. Now define a map $\sigma$ of $Y=\left(A_{0} \times\{0\}\right) \cup\left(B d\left(A_{1} \times l\right) \backslash \operatorname{Int}\left(A_{1} \times\{0\}\right)\right)$ into $E \backslash K$ by

$$
\sigma(x, t)= \begin{cases}\phi_{0}(x) & \text { if }(x, t) \in A_{0} \times\{0\} \\ b_{t}(x) & \text { if }(x, t) \in A^{\prime} \times\{t\} \\ \phi_{1}(x) & \text { otherwise. }\end{cases}
$$

It is easy to check that $\sigma$ is well defined and $p \sigma=\psi$. Since $E$ is trivial, $F$ an $\mathrm{AR}$ and $\sigma(Y) \cap K=\varnothing$, it follows that $\sigma$ can be extended to a map $\sigma_{1}$ of a neighborhood $V$ of $Y($ in $X)$ into $E \backslash K$ such that $p \sigma_{1}=\psi$.

Let $\lambda$ be an $A$-preserving imbedding of $A_{1} \times\{1\}$ into $V$ such that $\lambda(x, 1)=$ $(x, 0)$ for all $x \in A^{\prime}$. Define $\phi^{\prime}: A \rightarrow E \backslash K$ by

$$
\phi^{\prime}(x)= \begin{cases}\sigma_{1} \lambda(x, 1) & \text { when } x \in A_{1}, \\ \sigma_{1}(x) & \text { otherwise. }\end{cases}
$$

$\phi^{\prime}$ is the desired lifting. 
In our next lemma we let $E=(B \times F, p, B)$ be a product bundle over a Euclidean space (sufficiently large dimension) $B$ with fibre $F$ an $A R$ and let $K$ be a closed subset of $E$. We let $C_{n}$ denote a homeomorphic copy of $J^{n}\left(C_{0}\right.$ a single point). Let $\pi_{1}(n, k)$ denote the projection map of $C_{n} \times C_{k}$ onto $C_{n}$. We say $E$ satisfies property $P(n)$ if for any $C_{n} \subset B, k \geq 0$, every lifting of $\left.\pi_{1}(n, k)\right|_{\mathrm{Bd}\left(C_{n} \times C_{k}\right)}$ to $E \backslash K$ can be extended to a lifting of $\pi_{1}(n, k)$ to $E \backslash K$.

Lemma 2.5. If $E$ satisfies property $P(n)$ for some $n \geq 0$ then $E$ satisfies $P(n+1)$.

Proof. Let $k \geq 0$ be given. We regard $C_{n+1} \times C_{k}$ as $U_{t \in\}}\{t\} \times\left(C_{n} \times C_{k}\right)$ and let $\phi$ be a lifting of $\left.\pi_{1}(n+1, k)\right|_{\mathrm{Bd}\left(C_{n+1} \times C_{k}\right)}$ to $E \backslash K$. It follows from the hypothesis that, for each $t$, there is a map $\phi_{t}$ of $\{t\} \times C_{n} \times C_{k}$ into $E \backslash K$ such that $p \phi_{t}=\left.\pi_{1}(n+1, k)\right|_{\{t\} \times C_{n} \times C_{k}}$ and $\left.\phi_{t}\right|_{B d\left(\{t\} \times C_{n} \times C_{k}\right)}=\phi$. Since the fibre $F$ is an $\mathrm{AR}$ we can extend $\phi_{t}$ to a map $\phi_{t}^{\prime}$ of a neighborhood $V_{t}$ of $\{t\} \times C_{n} \times C_{k}$ into $E \backslash K$ satisfying $p \phi_{t}^{\prime}=\pi_{1}(n+1, k)$ and $\left.\phi_{t}^{\prime}\right|_{V_{t} \cap \mathrm{Bd}\left(C_{n+1} \times C_{k}\right)}=\phi$. It follows from compactness of $J$ that there are points $-1=a_{0}<a_{1}<\ldots<a_{n+1}=1$ in $J$ and maps $\left\{\phi_{i}^{*}\right\}_{i=0}^{n}$ such that for $A_{i}=\left[a_{i}, a_{i+1}\right] \times C_{n} \times C_{k^{\prime}}$ each $\phi_{i}^{*}: A_{i} \rightarrow E \backslash K$ satisfying $p \phi_{i}^{*}=\pi_{1}(n+1, k)$ and $\left.\phi_{i}^{*}\right|_{A_{i} \cap B d\left(C_{n+1} \times C_{k}\right)}=\phi$.

Let us consider $A_{0}, A_{1}$. At $A^{\prime}=A_{0} \cap A_{1}=\left\{a_{1}\right\} \times C_{n} \times C_{k}$, there are two maps $\left.\phi_{0}^{*}\right|_{A^{\prime}}$ and $\left.\phi_{1}^{*}\right|_{A^{\prime}}$ agreeing at $\operatorname{Bd}^{\prime}\left(A^{\prime}\right)$. Define $\psi: \operatorname{Bd}\left(\left\{a_{1}\right\} \times C_{n} \times C_{k} \times I\right)$ $\rightarrow E \backslash K$ by

$$
\psi\left(a_{1}, x_{1}, x_{2}, t\right)= \begin{cases}\phi_{0}^{*}\left(a_{1}, x_{1}, x_{2}\right) & \text { if } t<1, \\ \phi_{1}^{*}\left(a_{1}, x_{1}, x_{2}\right) & \text { otherwise. }\end{cases}
$$

$\psi$ is clearly a lifting of $\left.\pi_{1}(n, k+1)\right|_{\mathrm{Bd}\left(C_{n} \times C_{k+1}\right)}$ into $E \backslash K$, where $C_{k+1}=C_{k} \times I$. $E$ satisfies $P(n)$ implies that $\psi$ can be extended to a lifting of $\pi_{1}(n, k+1)$ to $E \backslash K$. It follows that $\left.\phi_{0}^{*}\right|_{A^{\prime}},\left.\phi_{1}^{*}\right|_{A^{\prime}}$ are $E$-homotopic in $E \backslash K$ modulo $\operatorname{Bd}\left(A^{\prime}\right)$. Hence, by Lemma 2.4, $\left.\pi_{1}(n, k+1)\right|_{A_{0} \cup A_{1}}$ can be lifted to a map $\phi_{1}^{\prime}$ of $A_{0} \cup A_{1}$ into $E \backslash K$ such that $\phi_{1}^{\prime}(x)=\phi_{i}^{*}(x)$ when $x \in \operatorname{Bd}\left(A_{0} \cup A_{1}\right) \cap A_{i}, i=0,1$. Next consider the pair $\left\{A_{1}, A_{2}\right\}$ and so on. It is clear that we can obtain the desired lifting.

Proof of Theorem 2.1. First we suppose $E$ is trivial and $(|K|,|L|)$ is the pair $(|\sigma|,|\dot{\sigma}|)$, where $|\sigma|$ is an $n$-simplex and $|\dot{\sigma}|=B \mathrm{~d}|\sigma|$. Let $E^{\prime}=\left(|\sigma| \times F, p^{\prime}\right.$, $|\sigma|)$ be the product bundle which is the pull-back of $E$ induced by $f$.

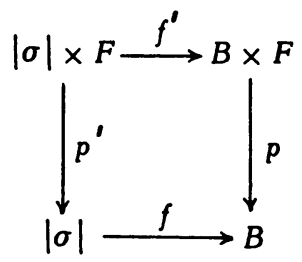


The lifting $\left(\left.f\right|_{\mid} \mid{ }^{*}\right)$ of $\left.f\right|_{\mid} \dot{\sigma}$ to $E \backslash A$ induces a continuous cross-section over $|\dot{\sigma}|$ in $E^{\prime}$, that is, a map $b:|\dot{\sigma}| \rightarrow(|\sigma| \times F) \backslash f^{\prime-1}(A)$ such that $p^{\prime} b=\mathrm{id}|\dot{\sigma}|$. If $b$ extends to a continuous selection $b_{1}$ of $|\sigma|$ into $(|\sigma| \times F) \backslash V^{\prime-1}(A)$, then it is clear that $f^{\prime} b_{1}$ is a desired lifting. To show $b_{1}$ exists we note that by hypothesis of $F$ and $A, E^{\prime}$ satisfies $P(0)$ as defined above. Lemma 2.5 implies that $E^{\prime}$ satisfies $P(n)$ for each $n$. Hence $b_{1}$ exists and the theorem is proved. For the general case we merely observe that by taking a finer triangulation, we may assume that, for each $\sigma \in K, E$ is trivial over $f(\sigma)$. We then construct the lifting inductively on $K_{i}$, the $i$-skeleton of $K$. By considering one simplex of $K_{i}$ at a time we reduce to the special case above.

3. Separation. Let $(E, p, B)$ be a bundle. We say $G: E \times I \rightarrow E$ is a bundle bomotopy provided each $g_{t}=\left.G\right|_{E \times\{t\}}$ is a bundle map; that is, $p g_{t}(x)=$ $p(x)$ for any $x \in E$. We say $G$ is a bundle isotopy provided in addition the induced map $E \times I \rightarrow E \times I$ is a homeomorphism. A homotopy $g_{t}: X \rightarrow Y$ is limited by an open cover $\mathcal{U}$ of $Y$ provided each orbit $\left\{g_{t}(x)\right\}_{t \in I}$ is contained in some member $U$ of $\mathcal{U}$. Let $A \subset X$ be closed and $\mathcal{U}$ be an open cover of $X \backslash A$. We say $U$ is normal (or normal with respect to $A$ ) if each map $f: X \backslash A \rightarrow X$ which is $\mathcal{U}$-close to the identity has an extension to $X$ which is the identity on $A$. Let $U \subset E$ be open, $E$ as above, and let $\mathcal{U}$ be any open cover of $\mathcal{U}$. Then by a $(U, \mathcal{U})$-isotopy $\left\{\mu_{t}\right\}$ we mean a bundle isotopy on $E$ such that each $\mu_{t}$ has support in $U$ (that is, $g_{t}$ is the identity outside $U$ ) and $\left\{\left.\mu_{t}\right|_{U}\right\}$ is limited by $\mathcal{U}_{\text {. Let }}$ $\mathcal{U}$ be a collection of subsets of a space $X$. We define the star of $\mathcal{U}$, denoted $\mathrm{St}(\mathcal{Y})$, to be the collection of all $V$ such that for some $U \in \mathcal{U}, V=\bigcup\{W \in \mathcal{U}$ : $W \cap U \neq \varnothing\}$. Inductively we define $\mathrm{St}^{n}(\mathcal{U})$ to be the star of $\mathrm{St}^{n-1}(\mathcal{U})$.

The main purpose of this section is to prove the following theorem.

Theorem 3.1. Let $(E, p, B)$ be a bundle over polybedron $B$ with fibre s. Suppose $A_{0}, A_{1}$ are closed sets in $E$ such that, for each $i=0,1, A_{i} \cap p^{-1}(b)$ is a Z-set in each $p^{-1}(b)$; then for any open neigbborbood $U$ of $A_{0}$ and any open cover $\mathcal{U}$ of $U$, there is a $(U, \mathcal{U})$-isotopy $\left\{\mu_{t}\right\}$ of $E$ such that $\mu_{0}=\mathrm{id}$ and $\mu_{1}\left(A_{0}\right)$ $\cap A_{1}=\varnothing$.

Moreover, the same is true if $A_{1}$ (not necessarily closed) is a countable union of closed sets $\bigcup_{i \geq 1} L_{i}$ sucb that, for each $i, L_{i} \cap p^{-1}(b)$ is a Z-set in each $p^{-1}(b)$.

The proof will be given at the end of the section.

The second part of Theorem 3.1 follows routinely from the first by applying the convergence procedure of Anderson-Bing [4].

By exact analogy from the proof of Theorem 3.1 we also have 
Theorem 3.2. Theorem 3.1 is true when fibre $=s$ is replaced by fibre $=Q$.

To give a proof of Theorem 3.1 we need several lemmas.

Lemma 3.3. Let $B$ be any metric space and let $K$ be a closed set in $E=$ $B \times Q$ of the bundle $(B \times Q, p, B)$. Then for any open cover $\mathcal{U}$ of $E$ there is a bundle bomotopy $\phi=\left\{\phi_{t}\right\}$ of $E$ into itself limited by U satisfying $\phi_{0}=\mathrm{id}$, $\phi_{1}(E)$ is closed in $E,\left.\phi_{t}\right|_{K}=$ id for eacb $t$ and $\phi_{1}(E \backslash K) \subset(B \times s) \backslash K$.

Remark. We actually construct $\phi$ so that each $\phi_{t}$ is an imbedding.

Proof. We write $Q$ as $\Pi_{i \geq 1} J_{i}, J_{i}=[-1,1]$. Let $O_{\text {be a locally finite open }}$ cover of $E \backslash K$ normal with respect to $K$ and such that $\mathcal{O}$ refines $\mathcal{U}$. Fix an $i \geq 1$. We write $Q$ as $Q_{i} \times J_{i}$ where $Q_{i}=\Pi_{k \neq i} J_{k}$ and let $E_{i}$ denote $B \times Q_{i}$. It is clear we can construct a cover 10 of $\left(E_{i} \times\{1\}\right) \backslash K$ such that each $W_{a} \in(\mathbb{B}$ has the form $W_{a}^{\prime} \times\left[t_{a}, 1\right], 1 / 3<t_{a}<1$, where $W_{a}^{\prime}$ is an open subset of $E_{i}$ and the collection $\left\{W_{a}^{\prime}\right\}$ is a locally finite refinement of $\mathcal{C}$. Define a function $f_{i}:\left(E_{i} \times\{1\}\right) \backslash K \rightarrow$ Reals by

$$
f_{i}((x, 1))=(1 / 3)\left(\max \left\{t_{\alpha}: x \in W_{a}^{\prime}\right\}+2\right)
$$

It is easily seen that $f_{i}$ is lower semicontinuous satisfying $1 / 3<f_{i}<1$. By Dowker's theorem (Dugundji, p. 171) there is a map $f_{i}^{\prime}:\left(E_{i} \times\{1\}\right) \backslash K \rightarrow$ Reals for which $f_{i}<f_{i}^{\prime}<1$. Hence, using normality of $\mathcal{C}$, there is a map $G_{i}: E_{i} \rightarrow$ Reals such that $1 / 3<G_{i}(x)<1$ whenever $(x, 1) \in\left(E_{i} \times\{1\}\right) \backslash K, G_{i}(x)=1$ whenever $(x, 1) \in K$ and each segment $\{x\} \times\left[G_{i}(x), 1\right]$ is contained in some member of $\mathcal{O}$. Similarly there is a map $g_{i}: E_{i} \rightarrow$ Reals such that $-1<g_{i}(x)<-1 / 3$ for any $(x,-1) \in\left(E_{i} \times\{-1\}\right) \backslash K, g_{i}(x)=-1$ for any $(x,-1) \in K$ and each segment $\{x\} \times$ $\left[-1, g_{i}(x)\right]$ is contained in some member of $\mathcal{O}$. Let $b_{i}, H_{i}: E_{i} \rightarrow$ Reals be maps satisfying $-1 \leq b_{i} \leq g_{i}, G_{i} \leq H_{i}<1$ and $-1<b_{i}(x)<g_{i}(x)$ whenever $(x,-1) \notin K$, $G_{i}(x)<H_{i}(x)<1$ whenever $(x, 1) \notin K$.

Denote by $\left\{\phi_{i t}\right\}$ the homotopy of $E_{i} \times J_{i}$ into itself such that for any $x \in E_{i}$, $\left\{\phi_{i t}\right\}$ moves $\{x\} \times J_{i}$ piecewise linearly onto $\{x\} \times\left[h_{i}(x), H_{i}(x)\right]$ while keeping $\{x\} \times\left[g_{i}(x), G_{i}(x)\right]$ pointwise fixed in the entire motion. Let $\phi$ denote the homotopy by applying $\left\{\phi_{1 t}\right\}$, which is followed by $\left\{\phi_{2 t}\right\}$, etc. Evidently $\phi$ exists and since at each step we may choose $\mathcal{C}$ to be arbitrarily small, we may require the homotopy to be limited by $U$. Finally we note that any bundle map of $B \times Q$ into itself is necessarily a closed map. $\phi$ is what we wanted.

The above construction also implies the following result of Chapman [9] which will be needed later. 
Lemma 3.4 (Chapman). Let $(B \times Q, p, B)$ be as above. Suppose $K$ is a closed subset of $B \times Q$ contained in $B \times s$; then $K$ is a Z -set of $B \times Q$ and there is an isomorphism of $B \times Q$ sending $K$ into $B \times Q_{1}$, where $Q_{1}=\Pi_{i \geq 1}[-1 / 2,1 / 2]_{i} \subset Q$.

The following lemma follows routinely from techniques of [6, Lemma 2.4].

Lemma 3.5. Let $M$ be an s-manifold and $(B \times M, p, B)$ a product bundle over metric space $B$. Let $X, Y$ be subsets of a complete separable metric space with $X$ closed. Suppose there is a map $f: X \cup Y \rightarrow B \times M$ sucb that $f(X \cup Y)$ is an M-projective Z-set; then for any open cover $\mathcal{U}$ of $\mathrm{cl}(f(X))$ there is a bomotopy $G=\left\{g_{t}\right\}$ of $X$ into $B \times M$ limited by $\mathcal{U}$ and satisfing the following conditions:

(1) $g_{0}=\left.f\right|_{X}$,

(2) $p G(\{x\} \times I)=\{$ point $\}$ for all $x \in X$,

(3) $G(X \times I)$ is an M-projective Z-set, and

(4) for all $t>0, g_{t}$ is an imbedding of $X$ onto a closed set in $B \times M$ such that $g_{t}(X) \cap f(X \cup Y)=\varnothing$.

The fact that any $s$-manifold $M$ is s-stable [7] and can be imbedded as an open subset of $s[12]$ are all that one needs to apply Lemma 3.3, Lemma 3.5 and the Anderson-McCharen [6] procedure to obtain the following homeomorphism extension lemma.

Lemma 3.6. Let $(B \times M, p, B)$ be as above. Let $G=\left\{g_{t}\right\}$ be a bomotopy of a complete metric space $A$ into $E=B \times M$ such that $g_{0}, g_{1}$ are imbeddings onto closed subsets of $E$. Suppose $G(A \times\{0,1\})$ is an M-projective Z-set and $p G(\{x\} \times I)=\{$ point $\}$ for eacb $x \in A$; then for any open neigbborbood $U$ of $\mathrm{cl}(G(A \times I))$ and any open cover $\mathcal{U}$ of $U$ by which $G$ is limited, there is a $\left(U, \mathrm{St}^{4}(\mathcal{U})\right.$ )-isotopy $\left\{\mu_{t}\right\}$ on $E$ such that $\mu_{0}=$ identity and $\mu_{1} g_{0}=g_{1}$.

Using Lemma 3.3 and the similar argument of Lemma 3.6 (by appealing to the techniques of Klee [13] and Anderson-McCharen) we can also conclude

Lemma 3.7. Lemma 3.6 is true if fibre $=Q$ and $A$ is compact metric. More. over, if $G(A \times\{0,1\}) \subset B \times s$, then we may choose $\left\{\mu_{t}\right\}$ to satisfy additionally that $\left\{\mu_{t \mid B \times s}\right\}$ is a $\left(U \cap s, \mathcal{U}_{1}\right)$-isotopy on $B \times s$, where $\mathcal{U}_{1}$ is the open cover of $U \cap s$ induced by restricting $\mathrm{St}^{4}(\mathrm{U})$ to $s$.

Lemma 3.8. Let $E^{\prime}=\left(B \times Q, p^{\prime}, B\right)$ and subbundle $E=(B \times s, p, B)$ be given over metric space $B$. Suppose $K$ is a closed subset of $B \times s$ such that $K \cap p^{-1}(b)$ is a $Z$-set in each $p^{-1}(b)$, and suppose $P$ is a compact polybedron in $B \times s$; then for any open set $U$ of $B \times Q$ containing $P$ and any open cover $U$ 
of $U$ in $B \times Q$, there is a $(U, \mathcal{U})-$ isotopy $\left\{\mu_{t}^{\prime}\right\}$ on $E^{\prime}$ sucb that $\left\{\mu_{t}=\left.\mu_{t}^{\prime}\right|_{B \times s}\right\}$ is a $\left(U \cap s, \mathcal{U} \cap s\right.$ )-isotopy on $E$ and $\mu_{1}(K) \cap P=\varnothing$.

Proof. We may assume $P=|T|$ for some finite simplicial complex $T$ and $\mathcal{U}$ is normal. Choose an open cover $\mathcal{O}$ of $|T|$ so that $\mathrm{St}^{5}(\mathcal{O})$ refines $\mathcal{U}$ and each $V \in \mathbb{C}$ has the form $V_{1} \times V_{2}$ where $V_{1}, V_{2}$ are open subsets of $B, Q$ respectively with $V_{2}$ convex. We may assume $|T|$ has been given a triangulation so that each simplex $\sigma \in T$ is contained in some $V \in \mathcal{C}$. Let $T_{i}$ denote the $i$-skeleton of $T$. For each $\sigma \in T$, let $V_{\sigma}=\bigcap\left\{V_{1} \times V_{2} \in \mathcal{Z}: \sigma \subset V_{1} \times V_{2}\right\}=\bigcap V_{1} \times$ $\cap v_{2}$. Note that $\cap v_{2}$ is convex. Let $f_{0}$ be any $B$-preserving map that carries $\left|T_{0}\right|$ into $(B \times s) \backslash K$ so that for each $\sigma \in T_{0}, f_{0}(\sigma) \in V_{\sigma}$. Now let $\sigma \in T_{1}$ and suppose $\sigma_{1}, \sigma_{2}$ are its vertices. By hypothesis of $V_{\sigma}, V_{\sigma} \supset\left(V_{\sigma_{1}} \cup V_{\sigma_{2}}\right)$ and $V_{\sigma}$ can be written as $V_{\sigma}(1) \times V_{\sigma}(2)$ with $V_{\sigma}(2)$ open and convex. By hypothesis of $K,\{x\} \times\left(V_{\sigma}(2) \cap s\right) \backslash\left(K \cap V_{\sigma}\right)$ is contractible (hence an AR) for each $x \in V_{\sigma}(1)$. Thus, by Theorem 2.1, $f_{0}$ can be extended to a $B$-preserving map $f_{1}$ of $\left|T_{0}\right| \cup \sigma$ into $(B \times s) \backslash K$ such that $f_{1}(\sigma) \subset V_{\sigma}$. This process clearly illus: trates that there is a $B$-preserving map $f$ of $|T|$ into $(B \times s) \backslash K$ such that $f(\sigma) C$ $V_{\sigma}$ for each $\sigma \in T$. Since each $V_{\sigma}(2) \cap s$ is convex, it follows that $f$ is homotopic to identity by a $B$-preserving homotopy $F$ of $|T|$ into $B \times s$ and is limited by 0 . Thus by Lemma 3.5 we may assume $f$ is an imbedding such that $f(|T|) \cap$ $K=\varnothing$ and $F$ limited by $S_{t}\left(\mathcal{C}^{0}\right)$. (Recall that $S_{t}{ }^{5}(\mathcal{O})$ refines $\mathcal{U}_{.}$)

It follows from Lemma 3.7 that there is a $\left(U, \mathrm{St}^{4}\left(\mathrm{St}_{\mathrm{t}}(\mathcal{O})\right)\right)$-isotopy $\left\{\lambda_{t}\right\}$ on $E^{\prime}$ such that $\left.\lambda_{1}\right|_{T}=1, \lambda_{t}(B \times s)=B \times s$ for all $t$ and $\left\{\lambda_{t}\right\}$ is limited by $S_{t}{ }^{5}(\mathcal{C})$. Then $\left\{\mu_{t}^{\prime}=\lambda_{t}^{-1}\right\}$ is a $\left(U, \mathcal{U}\right.$ )-isotopy on $E^{\prime}$ such that $\left\{\mu_{t}=\left.\mu_{t}^{\prime}\right|_{B \times s}\right\}$ is a $(U \cap s$, $\mathcal{U} \cap$ s)-isotopy on $E$ and $\mu_{1}(K) \cap|T|=\varnothing$.

Lemma 3.9. Let $E^{\prime}=\left(B \times Q, p^{\prime}, B\right)$ and subbundle $E=(B \times s, p, B)$ be given over compact polybedron $B$ and let $K$ be a closed set in $B \times$. s. Then the following are equivalent:

(1) $K \cap p^{-1}(b)$ is a Z.set in each $p^{-1}(b)$.

(2) There is an isomorpbism $\phi$ of $E^{\prime}$ such that $\phi(B \times s)=\phi(B \times s)$ and $\phi_{1}(K)$ is an s-projective Z.set, where $\phi_{1}=\left.\phi\right|_{B \times s^{*}}$.

Proof. $(2) \Rightarrow(1)$ is trivial. The proof of $(1) \Rightarrow(2)$ is essentially an application of Lemma 3.8, the technique used in proving [2, Lemma 7.1], and the results in [3]. We outline an argument in the following.

Let us define a standard $k$-cell in $s$ to be a set of the form $\Pi_{i=1}^{k}\left[a_{i}, b_{i}\right] \times$ $(0,0, \ldots)$ where $-1<a_{i}<b_{i}<1$ and $\left[a_{i}, b_{i}\right] \subset J_{i}$. Let $D$ denote a standard $k$-cell in $s$. It follows from Lemma 3.8 that for any open set $U$ of $B \times Q$ con- 
taining $B \times D$ and for any $\epsilon>0$, there is a $B$-preserving homeomorphism $/$ of $B \times Q$ onto itself supported in $U$ such that $f(B \times s)=B \times s, d(f$, id $)<\epsilon$ and $f(K)$ $\cap(B \times D)=\varnothing$.

$f$ is an exact analogue of the homeomorphism constructed in [2, Lemma 6.1] and was used to define $\left\{f_{\lambda}\right\}$ in the proof of $*$, where $*=$ Theorem 7.1 of [2]. In our case we denote such maps by $\left\{f_{\lambda}^{\prime}\right\}$. To define $b_{\lambda}^{\prime}$ as in the proof of $*$, we first let $\left\{b_{\lambda}\right\}$ be defined on $Q$ inductively using $\left\{f_{\lambda}^{\prime}\right\}$ as in $*$ and let $b_{\lambda}^{\prime}=$ id $_{B}$ $\times b_{\lambda}$. Let $g_{\lambda}^{\prime}=b_{\lambda}^{\prime} f_{\lambda}^{\prime}$. Then $g=\lim _{\lambda \rightarrow \infty} g_{\lambda}^{\prime} \cdots g_{2}^{\prime} g_{1}^{\prime}$ is a $B$-preserving homeomorphism of $B \times Q$ onto itself such that

$$
K_{0}=g(K) \cup \mathrm{cl}[g(B \times s) \cap(B \times(Q \backslash s))] \cup \mathrm{cl}[g(B \times(Q \backslash s)) \cap B \times s]
$$

has infinite partial deficiency (see [2]) with respect to $Q$. By applying Corollary 3.4 of [2], Lemma 5.1 of [3] and then Lemma 4.3 of [2] there is a B-preserving homeomorphism $b$ of $B \times Q$ onto itself such that $b g(B \times s)=B \times s$ and $b g(K)$ has infinite deficiency with respect to $Q . \phi=h g$ is a desired isomorphism. We remark that $\phi$ is obtained similarly to those constructed in [2, p. 378]. We simply observe, using the construction illustrated in [2] and results in [3], that we may choose $\phi$ to be $B$-preserving.

Lemma 3.10. Let $B$ be a polybedron or be as defined in Theorem 1.1. Then any bundle $E=(E, p, B)$ over $B$ with fibre $s$ is trivial.

Moreover, the same is true if the fibre is any metric space for which the structure group $G$ of the bundle $E$, when regarded as a subspace of the space of bomeomorphisms of fibre F (C.O topology), is contractible.

Proof of Lemma 3.10. First we suppose $B$ is a polyhedron. Let $\mathcal{U}=\left\{U_{a}\right\}$ denote an open cover of $B$ such that each $p^{-1}\left(U_{\alpha}\right)$ is trivial. We may assume $B=|K|$ for some lfsc $K$. Giving $K$ a finer triangulation if necessary we may assume each $\sigma \in K$ is already contained in some member of $\mathcal{U}$. Let $S_{i}, i \geq 0$, denote the $i$ th skeleton of $K$. Each $p^{-1}(|\sigma|), \sigma \in S_{0}$, is homeomorphic to $s$ and thus there is a bundle homeomorphism $b_{0}$ of $p^{-1}\left(\left|s_{0}\right|\right)$ onto $\left|S_{0}\right| \times s$. Since each 1-simplex is contained in some trivialization $p^{-1}\left(U_{a}\right)$, the set $p^{-1}(|\sigma|), \sigma \in S_{1}$, is homeomorphic to $|\sigma| \times s$. Using Theorem 2 of Renz [15] we can extend $b_{0}$ to a bundle homeomorphism of $p^{-1}\left(\left|S_{0}\right| \cup|\sigma|\right)$ onto $\left(\left|S_{0}\right| \cup|\sigma|\right) \times s$ for any $\sigma \in S_{1}$, hence to one of bundle $p^{-1}\left(\left|S_{1}\right|\right)$ onto $\left|S_{1}\right| \times s$. By analogy we then construct a sequence of bundle homeomorphisms $\left\{b_{n}\right\}, b_{n}: p^{-1}\left(\left|S_{n}\right|\right) \rightarrow\left|S_{n}\right| \times s$, such that $b_{n}$ extends $b_{n-1}$. An isomorphism for the lemma clearly follows.

The proof for the second half of the lemma is exactly the same. Finally if $B$ is not a polyhedron but given as in Theorem 1.1, then we simply observe that if $B C B^{\prime}$ is a retraction of some polyhedron $B^{\prime}$, then the pull-back bundle $E^{\prime}$ 
induced by a retraction $r: B^{\prime} \rightarrow B$ necessarily inherits the same structure group. Thus $E^{\prime}$ is trivial. But $E^{\prime}$ contains $E$; hence $E$ is also trivial.

If $F$ is a topological vector space (TVS) which is homeomorphic to $F^{\infty}$, by West [15A], $F$ has the reflective isotopy property. By Renz [15], $H(F)$ is contractible. Thus we have

Corollary 3.11. Let $B$ be as in Theorem 1.1. Then a bundle $(E, p, B)$ is trivial provided the fibre $F$ is a TVS homeomorpbic to $F^{\infty}$.

Similarly by Wong [16] and Renz [15], we have

Corollary 3.12. Let $B$ be as above. Then a bundle $(E, p, B)$ with fibre $Q$ is trivial.

Proof of Theorem 3.1. By Lemma 3.10 we may assume $E$ is the product bundle $(B \times s, p, B)$. First we consider the special case that $B$ is a compact polyhedron. By Lemma 3.9 we may assume $A_{0} \cup A_{1}$ is an s-projective Z-set. Let $\mathcal{C}^{\text {D }}$ be an open cover of $U$ such that $\mathrm{St}^{5}\left(\mathcal{C}^{\mathrm{O}}\right)$ refines $\mathcal{U}$. By Lemma 3.5 there is a homotopy $G=\left\{g_{t}\right\}$ of $A_{0}$ into $B \times s$ limited by $\mathbb{C}$ such that $g_{0}=$ id, $p G(\{x\} \times I)=$ point $\}$ for all $x \in A_{0}, G\left(A_{0} \times I\right)$ is a closed s-projective Z-set and $g_{1}$ is an imbedding for which $g_{1}\left(A_{0}\right) \cap A_{1}=\varnothing$. Now apply Lemma 3.6 to obtain a $\left(U, \mathrm{St}^{4}(\mathcal{Q})\right)$-isotopy $\left\{\mu_{t}\right\}$ on $E$ such' that $\left.\mu_{1}\right|_{A_{0}}=g_{1}$. Thus $\mu_{1}\left(A_{0}\right) \cap A_{1}=\varnothing$. But $\mathrm{St}^{5}(\mathcal{O})$ refines $\mathrm{U} ;\left\{\mu_{t}\right\}$ is a desired isotopy.

Now for the general case we may assume, by considering one component of $B$ at a time, that $B$ is connected. We of course can assume that $B$ is a lfsc $|K|$. Let $\sigma_{0}$ denote any simplex in $K$. Define $B_{1}=\bigcup\left\{\sigma \in|K|: \sigma \cap \sigma_{0} \neq \varnothing\right\}$. Inductively we define, for $n>1, B_{n+1}=\left(\bigcup\left\{\sigma \in|K|: \sigma \cap B_{n} \neq \varnothing\right\}\right) \backslash \operatorname{Int}\left(B_{n}\right)$. It follows from the assumptions on $B$ (local finiteness and connectedness) that each $B_{i}$ is a compact polyhedron, $B=\bigcup_{i \geq 1} B_{i}$ and $B_{i} \cap B_{j} \neq \varnothing$ only if $|i-j| \leq 1$.

By taking a normal open refinement of $\mathcal{U}$ if necessary we may assume. $U$ is normal.

Let $K_{0 j}=A_{0} \cap p^{-1}\left(B_{j}\right)$. Applying the special case proven above to each $K_{0 j}, j=$ odd, it follows that there is a $\left(U, \mathcal{U}_{0}\right)$-isotopy $\left\{\mu_{0 t}\right\}$ on $E$ such that $\mu_{01}\left(C_{1}\right)$ $\cap A_{1}=\varnothing$ where $\operatorname{St}\left(\mathcal{U}_{0}\right)$ refines $\mathcal{U}$ and $C_{1}=\bigcup_{j=0 d d} K_{0 j}$. Consider $\mu_{01}\left(C_{1}\right)$. Let $\mathcal{U}_{1}$ be a suitably chosen covering of $U$ such that $\mathcal{U}_{1}$ refines $\mathcal{U}_{0}$ and, for each $x \in \mu_{01}\left(C_{1}\right), \operatorname{cl}(V) \cap A_{1}=\varnothing$ whenever $x \in V, V \in \mathcal{U}_{1}$. Now applying the same process to $\left\{\mu_{01}\left(K_{0 j}\right)\right\}_{j=e v e n}$ there is a $\left(U, \mathcal{U}_{1}\right)$-isotopy $\left\{\mu_{1 t}\right\}$ on $E$ such that $\mu_{11}\left(C_{2}\right) \cap A_{1}=\varnothing$, where $C_{2}=\bigcup_{j=\text { even }} \mu_{01}\left(K_{0 j}\right)$. Define an isotopy $\left\{\mu_{t}\right\}$ on $E$ by letting $\left\{\mu_{t}\right\}_{0 \leq t \leq 1 / 2}$ be the isotopy $\left\{\mu_{0 t}\right\}_{t}$ (by an order preserving homeomorphism of $[0,1 / 2]$ onto $[0,1])$ and $\left\{\mu_{t}\right\}_{1 / 2 \leq t \leq 1}$ be the isotopy $\left\{\mu_{1} \mu_{01}\right\}_{t}$.

4. Mapping replacement. Let $\mathcal{U}$ be an open cover of a space $Y$. Maps $f, g$ : 
$X \rightarrow Y$ are $\mathcal{U}$-close provided that for each $x \in X$, there exists a $U \in \mathcal{U}$ for which $f(x), g(x) \in U$.

Theorem 4.1. Let $(E, p, B)$ be a bundle over polybedron $B$ with fibre s. Let $X$ be a separable complete metric space and $Y$ a closed set in $X$. Suppose $f: X \rightarrow E$ is a map such that $f(Y) \cap p^{-1}(b)$ is a Z-set in each $p^{-1}(b)$ and $f_{Y}$ is a closed imbedding; then for any open cover $\mathcal{U}$ of $\mathrm{cl}(f(X))$ there is a closed imbedding $g: X \rightarrow E$, U-closed to $f$, sucb that $g(X) \cap p^{-1}(b)$ is a Z-set in each $p^{-1}(b), p g=p f$ and $g$ extends $f_{Y^{\circ}}$

Moreover, the same is true when $B$ is a retract of a polyhedron.

Theorem 4.1 follows immediately from Theorem 1.1 and the following lemma. Before we state the lemma we note that a similar theorem for $Q$-bundles is true and may be concluded by exact analogy.

Theorem 4.2. Theorem 4.1 is true when fibre $=Q$ and $X$ is compact metric.

In the following lemma let $M$ denote an $s$-manifold.

Lemma 4.3. Let $(B \times M \times Q, p, B)$ be a bundle over space $B$. Let $X$ be a complete metric space and $Y \subset X$ be closed. Suppose there is a map $f$ of $X$ into $E=B \times M \times Q$ such that $f(X)$ is a Q-projective Z-set, $f(Y)$ is closed and $\Lambda_{Y}$ is an imbedding; then for any open cover $\mathcal{U}$ of $\mathrm{cl}(f(X))$ there is a bomotopy $\left\{g_{t}\right\}$ of $X$ into $E$ limited by U such that (1) $g_{n}=f$, (2) for all $t,\left.g_{t}\right|_{Y}=\Lambda_{Y}$ and $p g_{t}=p f$. (3) $g_{1}$ is an imbedding onto a closed Q-projective Z-set of $E$ and (4) $\left\{g_{t}\right\}$ is limited by $\mathrm{U}$.

Proof. The same as the mapping replacement lemma of [6].

Proof of Theorem 4.1. By Lemma 3.10 we may assume $E$ is the product oundle $(B \times s, p, \dot{B})$. The rest of the proof follows routinely from Lemmas 3.3, 3.4, Theorem 1.1 and Lemma 4.3.

5. Negligible subsets. Let $U$ be an open set in bundle $E=(E, p, B)$. Suppose $K \subset U$ is closed relative to $U$ and $U$ is an open cover of $U$; then by a $(U, \mathcal{U})$-extraction of $K$ from $E$ we mean an $I$-preserving homeomorphism

$$
G:(E \times I) \backslash(K \times\{1\}) \stackrel{\text { onto }}{\longrightarrow} E \times I
$$

such that for each $t, g_{t}=\left.G\right|_{E \times\{t\}}$ is a bundle map supported in $U, g_{0}=$ id and $\left\{\left.g_{t}\right|_{U}\right\}$ is limited by $U$. We say $K$ can be strongly extracted from $E$ provided that for each pair $(U, \mathcal{U})$ for which $K \subset U$ is closed relative to $U$, there is a $(U, \mathcal{U})$ extraction of $K$ from $E$. A subset $K \subset X$ is locally closed provided $K$ is closed relative to some open set which contains $K$. 
First we state a lemma which follows immediately from the technique of [10].

Lemma 5.1. Let $B$ be any space and let $K$ be a subset of $E=B \times M$ of bundle $(B \times M, p, B)$ with fibre $M$ an s-manifold. Suppose $K$ is a countable union of locally closed sets $K_{1}, K_{2}, \ldots$ such that each $K_{i}$ is an M-projective Z-set; then for any open cover $\mathcal{U}$ of $E$, there is an ( $E, \mathcal{U})$-extraction of $K$ from $E$.

Employing Theorem 1.1, Lemma 3.10 and Lemma 5.1 we obtain the following general statement of negligible subsets for bundles.

Theorem 5.2. Let $B$ be the same as in Theorem 1.1 and let $(E, p, B)$ be a bundle with fibre s. Suppose $A$ is a locally closed set in $E$ sucb that $\mathrm{cl}(A) \cap$ $p^{-1}(b)$ is a Z-set in each $p^{-1}(b)$; then $A$ can be strongly extracted from $E$.

Moreover, if instead $A$ is a countable union of locally closed sets, then for any open cover $\mathcal{U}$ of $E, A$ can be extracted from $E$ by an $(E, \mathcal{U})$-extraction.

The second half of Theorem 5.2 follows routinely from the first by employing an appropriate (but straightforward) refinement of the Anderson-Henderson-West techniques [5] together with the convergence procedure of Anderson-Bing [4].

6. Extending homeomorphism.

Theorem 6.1. Let $(E, p, B)$ be a bundle over polybedron $B$ with fibre $F=s$. Let $G=\left\{g_{t}\right\}$ be a bomotopy of a complete separable metric space $A$ into $E$ such that (i) $g_{0}, g_{1}$ are imbeddings onto closed sets in $E$, (ii) for $i=0,1, g_{i}(A) \cap$ $p^{-1}(b)$ is a Z-set in each $p^{-1}(b)$, and (iii) $p G(\{x\} \times I)=\{$ point $\}$ for each $x \in A$. Then there is an isotopy $\left\{\mu_{t}\right\}$ on $E$ such that $\mu_{1} g_{0}=g_{1}$. Moreover, if $U$ is an open neighborbood of $\mathrm{cl}(G(A \times I))$ and $\mathcal{U}$ is any open cover of $U$ for which $G$ is limited by ' $U$, we may choose $\left\{\mu_{t}\right\}$ to be an $\left(U, \mathrm{St}^{(4)}(U)\right)$-isotopy.

Furthermore, the same is true if $B$ is a retract of a polyhedron.

Proof. This is a simple consequence of Lemma 3.10, Theorem 1.1 and Lemma 3.6.

As a corollary we obtain an extension theorem which generalizes Theorem 2.1 in [17] and answers a question raised there.

Corollary 6.2. Let $\left(\Delta_{n} \times s, p, \Delta_{n}\right)$ be trivial over n-simplex $\Delta_{n}, n \geq 1$, and let $G=\left\{g_{t}\right\}$ be a bomotopy of a closed set $K_{1}$ in $\Delta_{n} \times s$ into $\Delta_{n} \times s$ such that $g_{0}=\mathrm{id}, g_{1}$ is an imbedding onto closed set $K_{2}, p G(\{x\} \times I)=\{$ point $\}$ for all $x$ and

$$
\left.g_{t}\right|_{\left(B d\left(\Delta_{n}\right) \times s\right) \cap K_{1}}=\mathrm{id}
$$

for all t. Suppose, for $i=1,2, K_{i} \cap p^{-1}(b)$ is a Z.set in $p^{-1}(b)$ for each $b \epsilon$ $\operatorname{Int}\left(\Delta_{n}\right)$; then for any open cover $\mathcal{U}$ of $\Delta_{n} \times s$ by which $G$ is limited, there is a 
$\left(\Delta_{n} \times s, \mathrm{St}^{(4)}(\mathcal{U})\right) \cdot$-isotopy $\left\{\mu_{t}\right\}$ on $\Delta_{n} \times s$ such that $\mu_{0}=$ identity, $\left.\mu_{1}\right|_{K_{1}}=g_{1}$ and $\left.\mu_{t}\right|_{\mathrm{Bd}\left(\Delta_{n}\right) \times s}=\mathrm{id}$ for all $t$.

As another application we obtain the following isotopy lemma. For a subset $A$ of any space $X$, let $H_{A}(X)$ denote the space of all homeomorphisms (compactopen topology) of $X$ leaving $A$ pointwise fixed.

Corollary 6.3. Let $A$ be a closed Z-set in s. Then $H_{A}(s)$ is bomotopically trivial.

It is known that, for $A=\varnothing, H_{A}(s)$ is contractible [15]. W. Barit in [8] has shown that in general the first homotopy group of $H_{A}(s)$ is trivial and in fact, for $X=s$ or $Q, H_{A}(X)$ is contractible provided $A$ is compact. Thus we ask the following:

Open question. Is $H_{A}(s)$ contractible?

Corollary 6.3 is an immediate consequence of Corollary 6.2. For if $f$ : $\operatorname{Bd}\left(\Delta_{n}\right) \rightarrow H_{A}(s)$ is a map we can interpret $f$ as an isomorphism of the bundle $\operatorname{Bd}\left(\Delta_{n}\right) \times s=\left(\operatorname{Bd}\left(\Delta_{n}\right) \times s, p, \operatorname{Bd}\left(\Delta_{n}\right)\right)$ such that $\left.f\right|_{\operatorname{Bd}\left(\Delta_{n}\right) \times A}=$ id. By Renz [15] or Wong [16] we can extend $f$ to an isomorphism $F$ on the bundle $\Delta_{n} \times s=\left(\Delta_{n} \times s\right.$, $p, \Delta_{n}$ ). We then use Corollary 6.2 to obtain another isomorphism $F_{1}$ on $\Delta_{n} \times s$ such that $\left.F_{1}\right|_{B d\left(\Delta_{n}\right) \times s}=$ id and $\left.F_{1}\right|_{\Delta_{n} \times A}=\left.F\right|_{\Delta_{n} \times A^{*}} F_{1}^{-1} F$ is a required extension of $f$ to $\Delta_{n} \times s$.

By analogy we have

Theorem 6.4. Theorem 6.1 is true if fibre $=Q$ and $A$ is compact metric.

Proof. This follows from Corollary 3.12, Theorem 3.10 and Lemma 3.7.

Corollary 6.5. Corollary 6.2 is true if fibre $=Q$.

From this we can conclude a corollary similar to Corollary 6.3, and give a different proof ( $a$ weaker version) of the result by Barit.

Corollary 6.6. $H_{A}(Q)$ is bomotopically trivial provided $A$ is a closed Z-set in $Q$.

\section{REFERENCES}

1. R. D. Anderson, Hilbert space is homeomorphic to the countable infinite product of lines, Bull. Amer. Math. Soc. 72 (1966), 515-519. MR $32 \# 8298$.

2. - On topological infinite deficiency, Michigan Math. J. 14 (1967), 365-383. MR 35 \# 4893.

3. - Topological properties of the Hilbert cube and the infinite product of open intervals, Trans. Amer. Math. Soc. 126 (1967), 200-216. MR 34 \# 5045.

4. R. D. Anderson and R. H. Bing, A complete elementary proof that Hilbert space is homeomorphic to the countable infinite product of lines, Bull. Amer. Math. Soc. 74 (1968), 771-792. MR 37, \#5847. 
5. R. D. Anderson, David W. Henderson, and James E. West, Negligible subsets of infinite-dimensional manifolds, Compositio Math. 21 (1969), 143-150. MR 39 \#7630.

6. R. D. Anderson and John D. McCharen, On extending homeomorphisms to Fréchet manifolds, Proc. Amer. Math. Soc. 25 (1970), 283-289. MR 41 \#2711.

7. R. D. Anderson and R. M. Schori, Factors of infinite-dimensional manifolds, Trans. Amer. Math. Soc. 142 (1969), 315-330. MR 39 \#7631.

8. W. Barit, Some properties of certain subsets of infinite-dimensional spaces, Dissertation, Lousiana State University, 1971.

8A. C. Bessaga and V. Klee, Every non-normable Fréchet space is homeomorphic with all its closed convex bodies, Math. Ann. 163 (1966), 161-166. MR 34 \#1826.

9. T. A. Chapman, Four classes of separable metric infinite-dimensional manifolds, Bull. Amer. Math. Soc. 76 (1970), 399-403. MR $40 \# 6590$.

9A. - Infinite deficiency in Fréchet manifolds, Trans. Amer. Math. Soc. 148 (1970), 137-146. MR $41 \# 1074$.

10. W. H. Cutler, Negligible subsets of infinite-dimensional Fréchet manifolds, Proc. Amer. Math. Soc. 23 (1969), 668-675. MR 40 \#2133.

11. J. Eells, Jr. and N. H. Kuiper, Homotopy negligible subsets, Compositio Math. 21 (1969), 155-161. MR $40 \# 6546$.

12. D. W. Henderson, Infinite-dimensional manifolds are open subsets of Hilbert space, Bull. Amer. Math. Soc. 75 (1969), 759-762. MR 40 \#898.

12A. D. Husemoller, Fibre bundles, McGraw-Hill, New York, 1966. MR 37 \#4821.

13. V. L. Klee, Convex bodies and periodic homeomorphisms in Hilbert space, Trans. Amer. Math. Soc. 74 (1953), 10-43. MR 14, 989.

14. R. S. Palais, Homotopy theory of infinite dimensional manifolds, Topology 5 (1966), 1-16. MR 32 \#6455.

15. P. Renz, The contractibility of the homeomorphism group of some product spaces by Wong's method, Math. Scand. 28 (1971), 182-188.

15A. J. E. West, Fixed point sets of transformation groups on infinite-product spaces, Proc. Amer. Math. Soc. 21 (1969), 575-582. MR 39 \#945.

16. R. Y. T. Wong, On homeomorphisms of certain infinite dimensional spaces, Trans. Amer Math. Soc. 128 (1967), 148-154. MR 35 \# 4892.

17. - Stationary isotopies of infinite-dimensional spaces, Trans. Amer. Math. Soc. 156 (1971), 131-136. MR 43 \#1230.

18. - Homotopy negligible subsets of bundles, Compositio Math. 24 (1972), 119-128.

19. T. A. Chapman and R. Y. T. Wong, On homeomorphisms of infinite dimensional bundles. II, Trans. Amer. Math. Soc. 191 (1974), 261-268.

20. - On homeomorphisms of infinite dimensional bundles. III, Trans. Amer. Math. Soc. 191 (1974), 269-276.

DEPARTMENT OF MATHEMATICS, UNIVERSITY OF CALIFORNIA, SANTA BARBARA, CALIFORNIA 93106 